\title{
Protein structure-based drug design: applications, limitations and future developments
}

\section{Alexander Hillisch}

Address: Bayer HealthCare AG, Director, Medicinal Chemistry, 40296 Wuppertal, Germany

Email: Alexander Hillisch - alexander.hillisch@bayerhealthcare.com

from 3rd German Conference on Chemoinformatics

Goslar, Germany. II-I3 November 2007

Published: 26 March 2008

Chemistry Central Journal 2008, 2(SuppI I):SI5 doi:I0.II86/1752-I53X-2-SI-SI5

This abstract is available from: http://www.journal.chemistrycentral.com/content/2/SI/SI5

(c) 2008 Hillisch

Protein structure-based drug design has been contributing to the drug discovery process since the early 1990s. Structural knowledge on the exact interactions of drugs with their target proteins has been applied mainly to predict potency changes of chemically modified lead compounds.

With the 3D-structural information available today, additional aspects of the drug discovery process become predictable. Target-based virtual screening is being applied to identify new unexpected lead structures. Selectivity of compounds between homologous or orthologous proteins can be predicted, offering new possibilities to design selective compounds or predict the suitability of animal models for pharmacodynamic studies. Also the number of $\mathrm{x}$-ray structures of proteins relevant for ADMET properties of drug molecules has remarkably increased during the last few years. This development offers the possibility to modulate or rationally design out unwanted ADMET properties. This covers aspects such as plasma protein binding, cytochrome P450 inhibition, metabolism and induction.

In this review style talk, a number of recent examples will be presented that demonstrate the utility of protein structure information during the drug discovery process, from lead identification to lead optimization and from pharmacodynamics to pharmacokinetic studies. 